\title{
Potencial para criação de empreendimentos de base tecnológica com os conhecimentos gerados em pesquisa
}

\author{
Marcelo Farid Pereira* \\ Marcela Gimenes Bera
}

\begin{abstract}
RESUMO - Existe nas universidades brasileiras um significativo acervo de pesquisas que representa um potencial de inovação empresarial e de criação de empreendimentos de base tecnológica. Entretanto, para que isso aconteça se faz necessário o atendimento de algumas condições, como relação com mercado, exequibilidade técnica e infraestrutura de apoio. Sendo assim, esse estudo tem como objetivo verificar as possibilidades de pesquisas se transformarem em negócios. Dessa forma, foi realizado um estudo no Departamento de Engenharia Química da Universidade Estadual de Maringá, que possui pesquisa significativa nos cursos de Mestrado e Doutorado, para verificar o potencial de criação de empreendimentos de base tecnológica com os conhecimentos gerados nos projetos de pesquisa. O estudo procurou caracterizar, dentre outros fatores, os aspectos gerais da pesquisa: as dificuldades no desenvolvimento, o potencial do produto pesquisado, registro de patente e a relação com o mercado. Através da metodologia aplicada constatou-se que há potencial de criação de empreendimentos de base tecnológica, pois a maioria das pesquisas que estão sendo desenvolvidas pelos docentes tem por finalidade a geração de patentes e criação de produtos.
\end{abstract}

Palavras-chave: Inovação tecnológica. Transferência de tecnologia. Criação de empresas de Base Tecnológica.

\section{INTRODUÇÃO}

As universidades brasileiras podem difundir e transferir conhecimentos devido ao seu grande acervo de pesquisas, pois, segundo Feldman (1994 a, citado por Rapine, 2000), o processo de inovação depende de conhecimento mais do que qualquer outra atividade econômica. Assim, um maior estreitamento entre as universidades e o setor produtivo pode ser uma alternativa de difusão de novas tecnologias e novos negócios.

Neste sentido, a geração de novas tecnologias dentro das universidades e as possíveis parcerias com o setor produtivo se tornaram importantes para o desenvolvimento do país, contribuindo, para o aumento da competitividade.

\footnotetext{
* Doutor em Engenharia de Produção - Universidade Federal de Santa Catarina (1999) Professor Associado do Departamento de Economia da Universidade Estadual de Maringá; Coordenador do Grupo de Apoio Estratégico da Incubadora Tecnológica de Maringá. Endereço eletrônico: mfpereira@uem.br.

** Graduada em Ciências Econômicas - Universidade Estadual de Maringá (2008). Economista do Grupo de Apoio Estratégico da Incubadora Tecnológica de Maringá. Endereço eletrônico: beragimenes@bol.com.br.
} 
De acordo com Castro (2006), o conhecimento passou a ter um papel central no desenvolvimento econômico, sendo assim o principal fator no processo de inovação e aumento da competitividade. A base industrial do Brasil historicamente foi consolidada na importação maciça de tecnologias, gerando diversos problemas para o desenvolvimento nacional, principalmente na área do conhecimento.

Depois da década de 90, com a abertura comercial, o país vem buscando uma maior competitividade, e esta capacidade de geração de conhecimento interno passa a ser fundamental para o desenvolvimento do país. O grande problema dos países em desenvolvimento é a carência de recursos disponíveis para os investimentos em pesquisas, visto que possuem um risco e retorno de longo prazo sendo este um limitador na captação de financiamento.

Assim, torna-se importante a realização de estudos que tratem do potencial de desenvolvimento de novas tecnologias, decorrentes de pesquisas realizadas por instituições de ensino e pesquisa, que possam ser transferidas para o setor produtivo para inovação empresarial e criação de novos empreendimentos de base tecnológica.

Neste contexto, considera-se relevante verificar qual o potencial de transformação de Projetos de Pesquisa do Departamento de Engenharia Química da Universidade Estadual de Maringá, o qual possui curso de mestrado e doutorado e pesquisa significativa em inovação empresarial e na criação de negócios de base tecnológica.

\section{TRANSFERÊNCIA TECNOLÓGICA}

Bessant e Rush (1993 apud França, 2001) definem transferência de tecnologia como um conjunto de processos e atividades no qual a tecnologia é passada de um usuário a outro, incorporada em novos processos e produtos, ou desincorporada em formas como conhecimento, habilidades e direitos legais.

Segundo Roman (1983, apud França, 2001), o processo de disseminação com sucesso da informação técnica e científica a um recebedor através de mecanismos formais e informais é transferência tecnológica.

Já para Marchiori e Colenci Junior (2000), a transferência de tecnologia é um processo de transferência de informação e de capacidades específicas, desenvolvidas através de pesquisa, que tem por fim gerar capacitação tecnológica das empresas receptoras.

A transferência de tecnologia pode ser horizontal ou vertical. Segundo Cleto (1996), a pesquisa, realizada em laboratório, no desenvolvimento de um produto ou processo 
produtivo, classifica-se como transferência tecnológica vertical. A transferência tecnológica horizontal é a transferência de conhecimento estabelecido, ou de processos de um ambiente para outro, de modo a aproveitar a capacitação desenvolvida, ou seja, é o melhoramento de um produto já existente no mercado.

Segundo Rapine (2007), o moderno papel da informação e do conhecimento nas economias e no processo produtivo têm instigado um reposicionamento do papel desempenhado pelas universidades, as quais não apenas são responsáveis pelo treinamento, mas também por fornecer conhecimento crucial para o enriquecimento de alguns setores industriais.

Transferência de tecnologia é a condução de um conjunto de conhecimentos e práticas tecnológicas de uma instituição para outra, incluindo as diversas fases componentes do processo (NETO, 1983, p. 362 apud MARCHIORI e JUNIOR, 2000).

Pode-se observar na Figura 1 alguns integrantes de geração de tecnologia. A empresa produtora está diretamente ligada à Pesquisa e Desenvolvimento e possui relacionamento com a universidade, o fabricante de equipamentos, o instituto de pesquisa aplicada e a empresa de engenharia. Também se observa a interação com o mercado.

\section{FIGURA 1 - ALGUNS INTEGRANTES NA GERAÇÃO E TRANSFERÊNCIA DE TECNOLOGIA}

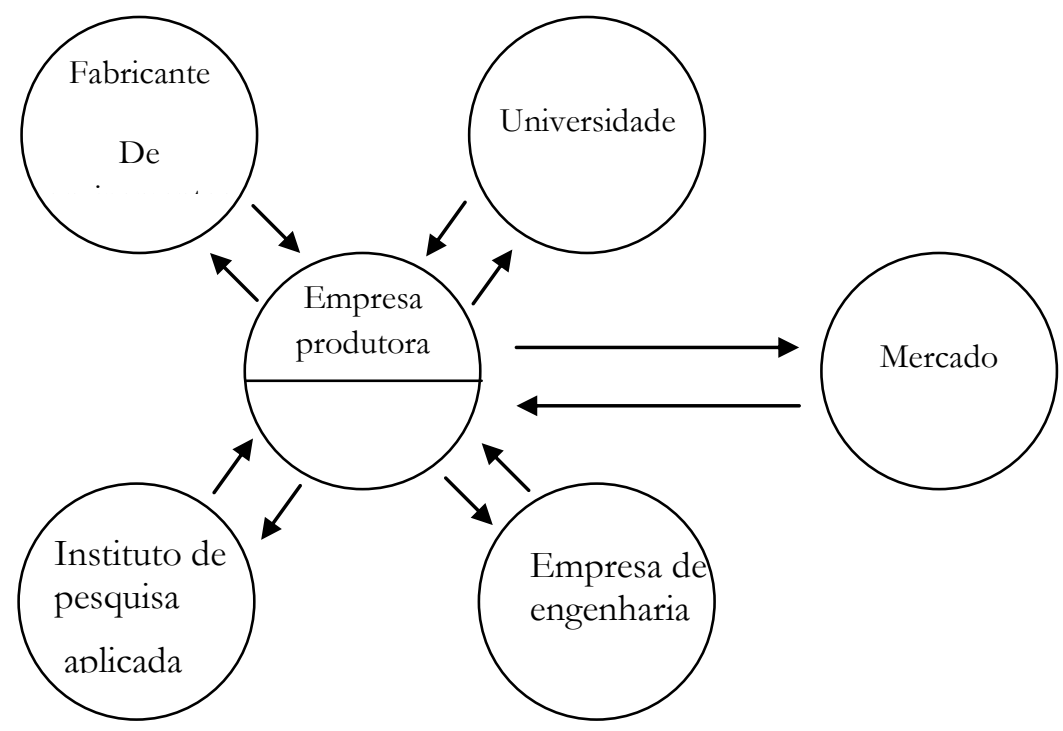

FONTE: MARCHIORI e JUNIOR apud (NETO 1983).

Segundo Ribeiro (2001), diversos países já estão utilizando determinados modelos de transferência de tecnologia como: incubadora de empresas, parques tecnológicos e aglomerados produtivos. 
Portanto, a transferência de tecnologia é o processo no qual o resultado de pesquisa básica aplicada e desenvolvimentos experimentais são administrados pela instituição de pesquisa ou empresas para o setor industrial e comercial. Onde ocorre troca de informações entre os pesquisadores que criam a inovação tecnológica e os usuários da idéia nova.

Segundo Longo e Oliveira (1995) desde o início dos anos 70, em vários países industrializados, houve diversas tentativas para diminuir os custos das pesquisas, onde deram razão ao aparecimento de formas para o desenvolvimento tecnológico, denominada, de forma genérica, de pesquisas cooperativas.

Antes de 1980, conforme Ben (2007), houve um licenciamento esporádico de transferência tecnológica em determinadas universidades inglesas e americanas. A partir de 1980 a transferência de tecnologia nos EUA era bem esporádica e complexa, e raramente recompensadora. Nesta mesma década houve a aprovação da Lei Bayh-Dole, onde se criou uma política de criação de patentes, dando às universidades e aos pesquisadores o direito de propriedade de patente com exclusividade, quando tenham sido desenvolvidos com recursos governamentais.

A Lei de Bayh-Dole foi, sobretudo, um encorajador instrumental para as universidades participarem de atividades relacionadas transferência tecnológica. Pois as instituições de ensino são incentivadas a colaborar com preocupações comerciais, promovendo assim a utilizações das invenções resultantes do financiamento federal ${ }^{60}$.

A partir de 1990, com a dinamização da economia, vários países promulgaram o Bayh-Dole, iniciando a transferência de tecnologia da universidade para a indústria, entre eles, Alemanha, Japão, Cingapura, Hong Kong, Taiwan, Brasil, China e África do Sul.

De acordo com Ben (2007), a partir de 2000 o interesse em transferência tecnológica é mundial. Países começaram a procurar inovações tecnológicas em suas universidades, na óptica da "Economia do conhecimento", ou seja, intenso interesse em ampliar capacitações e acelerar a aprendizagem e o treinamento.

Segundo Póvoa (2006), a atividade de patenteamento por parte das universidades brasileiras é contemporânea. Onde o primeiro registro de patente em nome de uma universidade foi em $1979^{61}$ (Universidade Federal do Rio de Janeiro). Entretanto, é provável que antes desta data pesquisadores dentro das universidades, tenham requerido pedidos em seu nome.

\footnotetext{
${ }^{60}$ Mais de $90 \%$ das pesquisas que se desenvolve nos EUA são com recursos governamentais. ${ }^{61}$ Obteve a carta de patente somente em 1985.
} 
Pode se observar no Gráfico 1 a evolução de patentes de universidades brasileiras entre 1979-2004.

GRÁFICO 1 - EVOLUÇÃO DOS DEPÓSITOS DE PATENTES DE UNIVERSIDADES BRASILEIRAS 1979-2004

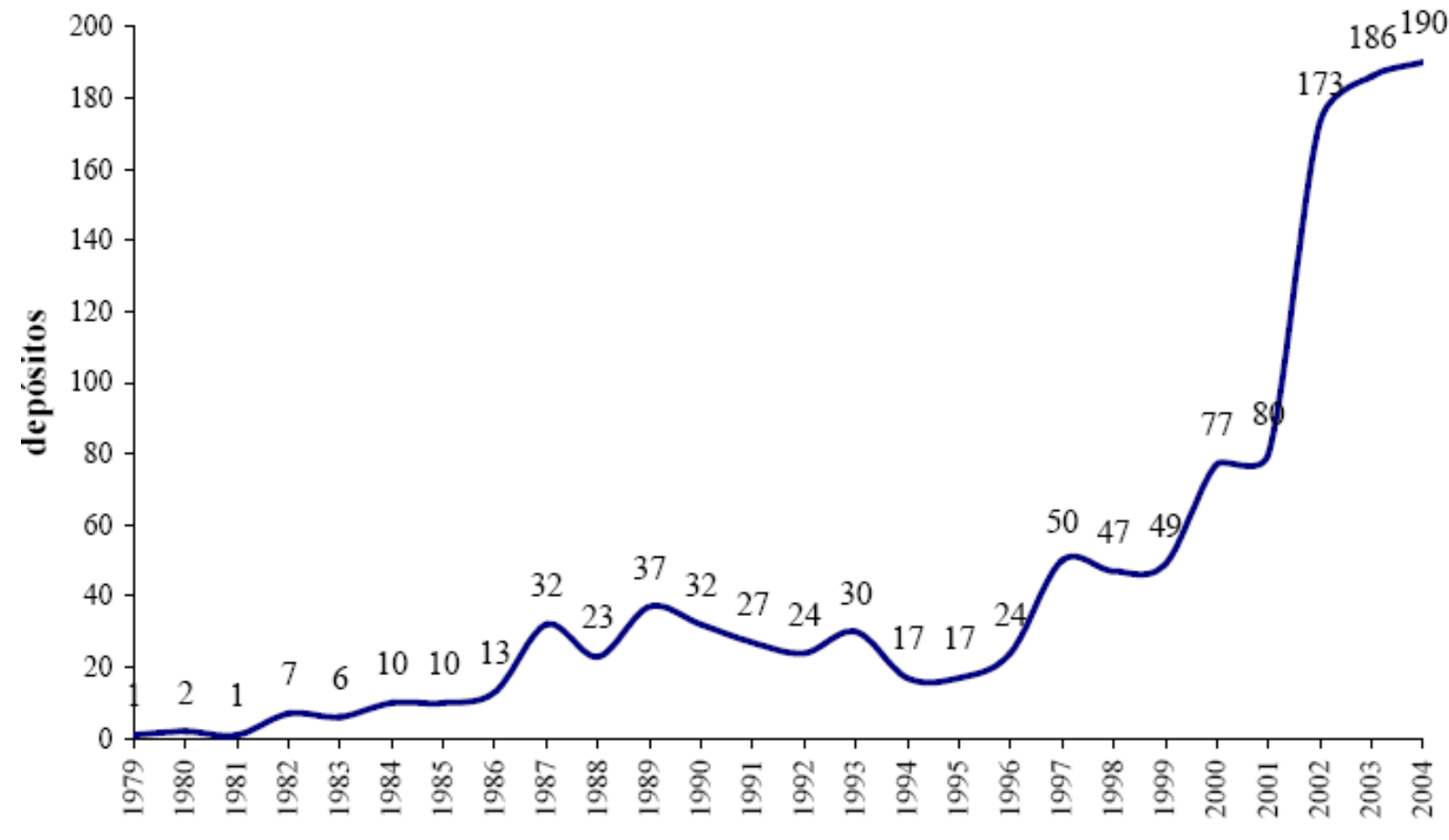

FONTE: POVOA, 2006.

Segundo Povoa (2006), este aumento nos depósitos de patentes a partir de 1997 deve-se à nova lei de propriedade industrial (Lei no 9.279 de 14 de maio de 1996) que substituiu a Lei $n^{\circ} 5.772$ de 21 de dezembro de $1971^{62}$.

\section{METODOLOGIA}

Foi aplicado um questionário aos Docentes do Departamento de Engenharia Química da Universidade Estadual de Maringá. É importante destacar que os questionários foram repassados a 28 professores, porém apenas 6 responderam, o que representa um limitador da pesquisa. Entretanto, uma nova pesquisa junto a todo Centro Tecnológico da UEM, está em andamento, com medidas para obter um maior número de questionários respondidos.

62 Antes de maio de 1997, não era patenteável a invenção relacionada com medicamentos fármacos. Com a criação da Lei $n^{\circ} 9.279$ (Lei da Propriedade Industrial), permitiu-se a proteção da patente, dando o direito ao inventor obter uma patente que garantisse a propriedade pela invenção. 
A pesquisa foi realizada do dia 15 de novembro de 2007 ao dia 2 de abril de 2008, com 16 perguntas na maioria fechadas e múltiplas, e dividida em quatro partes: na primeira limitou-se em conhecer alguns aspectos gerais do projeto, como há quanto tempo estão sendo desenvolvidos; na segunda parte, foram abordadas as questões de dificuldades no desenvolvimento; na terceira procurou-se conhecer a motivação para o desenvolvimento; e na quarta parte tentou-se observar o potencial do produto pesquisado, registro de patente e relação com o mercado, que é o tema central da pesquisa.

\section{RESULTADOS}

Com base nas informações coletadas na primeira parte do questionário, observou-se que a maioria das pesquisas tem aproximadamente 5 participantes e que 33\% delas estão sendo realizadas entre dois e quatro anos, e 67\% há mais de quatro anos. Isso demonstra que existe participação expressiva de pessoal e tempo nos projetos.

A segunda parte está representada no Gráfico 2, onde podem ser observadas as principais dificuldades no desenvolvimento das pesquisas. O primeiro fator é a falta de financiamento, e o segundo é a falta de infraestrutura, e em terceiro é a inovação.

GRÁFICO 2 - DIFICULDADES NO DESENVOLVIMENTO DA PESQUISA

\section{口Outros}

口 Inovaçã̃o

口 Infra estrutura

Financiamento

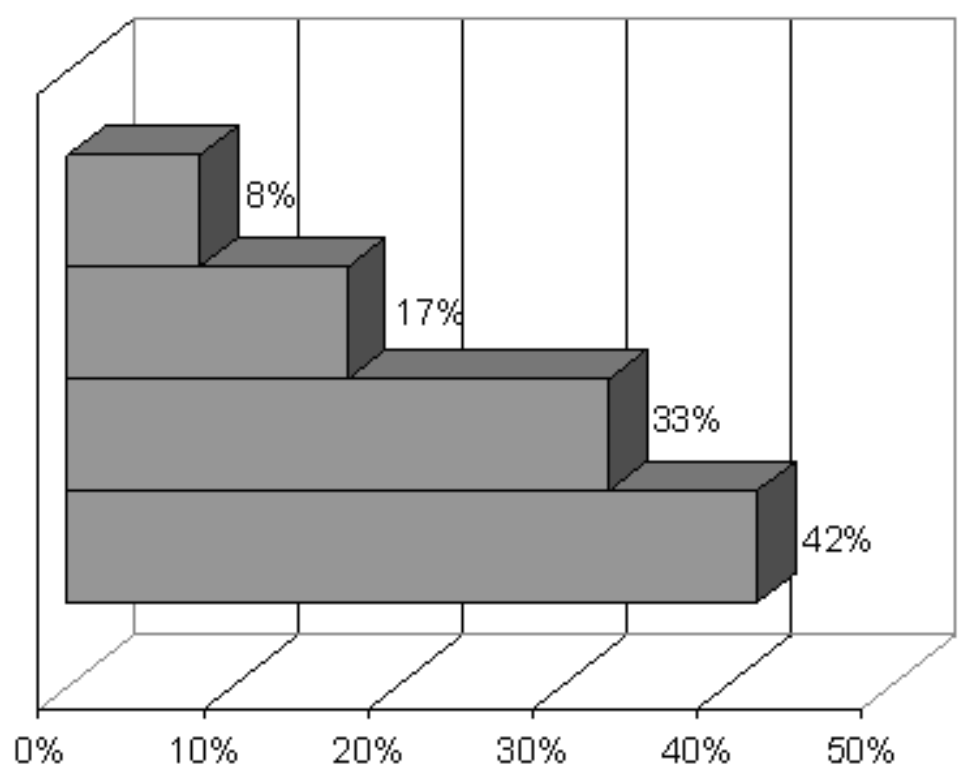

FONTE: elaboração própria.

A motivação observada na terceira parte estava mais relacionada à inovação e orientação, onde se observou que $87 \%$ têm por objetivo a inovação tecnológica em conjunto com orientação, e 17\% tem objetivo apenas de orientação. O que representa uma possibilidade com interação com mercado. 
Por fim, na quarta parte, que trata do potencial de inovação tecnológica e criação de empreendimentos inovadores, foi observado que 33\% das pesquisas já têm registro de patente e $67 \%$ ainda não. Entretanto, dentre os docentes que não tem registro de patente na sua pesquisa $83 \%$ deles pretende fazer, e $17 \%$ não respondeu sobre esse aspecto. Ainda foi constatado que $100 \%$ dos docentes acreditam que sua pesquisa tem potencial de se transformar em empreendimento de base tecnológica.

Por outro lado é importante destacar que, apesar dos docentes acreditarem no potencial de seus produtos, eles não realizaram pesquisas de mercado ou plano de negócio. Vale relatar que $67 \%$ dos pesquisadores têm interesse em fazer um plano de viabilidade econômica.

A realização de uma pesquisa de mercado ou plano de negócio é importante para direcionar ações em relação a produtos.

Por fim cabe ainda destacar que 67\% dos docentes pesquisados desconhecem o processo de incubação de empresas, mesmo sendo a Incubadora Tecnológica de Maringá situada dentro da Universidade (UEM).

\section{CONSIDERAÇÕES FINAIS}

Com base nos resultados pode-se observar que as pesquisas têm um significativo número de pessoal envolvido e de tempo despendidos em seu desenvolvimento. No caso das dificuldades observou-se a carência de infra-estrutura e financiamento. Já a motivação para a pesquisa parece estar bem direcionada, além da formação acadêmica, para a aplicação empresarial. Corrobora com isso quando observa-se que 33\% das pesquisas já têm registro de patente e a maioria restante pretende fazer, representando um potencial para inovação. Porém vale destacar que esse potencial será maximizado quando as patentes antes de serem requeridas, passarem por um conselho técnico, que dê um parecer preliminar, sobre viabilidade técnica e econômica. No caso da UEM esse processo é adotado.

De um modo geral, observou-se, que estão sendo realizadas pesquisas com potencial de aplicação em atividades produtivas e que podem vir a contribuir para melhorar a competitividade do sistema produtivo nacional. Porém, fica evidente também a carência de mecanismos de interação com o setor produtivo. 


\section{REFERÊNCIAS}

BEM, Renée Israel. A Promessa de Transferência Tecnológica Universitária. X Repicit. Rio de Janeiro, Agosto de 2007. Adaptado com permissão de Lita Nelsen - MIT. Disponível $\mathrm{em}:<$ www.redetec.org.br/publique/media/x repict renee ben israel.pdf $>$. Acesso em $27 / 02 / 2008$.

CASTRO, Alexandre Camargo. Produção e disseminação de informação tecnológica: a atuação da INOVA - Agência de Inovação da Unicamp. Dissertação (mestrado) Pontifícia Universidade Católica de Campinas, Centro de Ciências Sociais Aplicadas, PósGraduação em Ciência da Informação. Disponível em:

$<\underline{\text { http://dissertacaoinova.tripod.com/sitebuildercontent/sitebuilderfiles/producao e dissemi }}$ nacao de informacao tecnologica.doc $>$. Acesso em 21/02/2008.

CLETO, Marcelo G. Proposta de Estruturação da Transferência de Tecnologia IntraFirma para Produção no Exterior em Empresa Brasileira: O Caso do Metal Leve S.A. Tese de doutorado Cap.3 A Questão da Transferência de Tecnologia e da Produção Internacional. Disponível em:

< http://www.eps.ufsc.br/teses96/cleto/cap3/cap3.htm>. Acesso em 13/04/2008.

POVOA, Luciano Martins Costa. Depósitos de Patentes De Universidades Brasileiras (1979-2004). Disponível em:

$<\underline{\text { http://www.cedeplar.ufmg.br/seminarios/seminario diamantina/2006/D06A006.pdf }>}$

Acesso em 15/03/2008.

LONGO, Waldimir Pirró e OLIVEIRA, Antônio Ricardo Pimentel de. Pesquisa Coperativa e Centros de Excelência. Disponível em:

<http://www.waldimir.longo.nom.br/artigos/95.doc>. Acesso em: 12/03/2008.

MARCHIORI, M. P.; COLENCI JUNIOR, Alfredo. Transferência de Tecnologia Universidade-Empresa - A Busca por Mecanismos de Integração Efetiva. Revista de Ciência e Tecnologia, Política e Gestão para a Periferia. RECITEC, Recife, v.4, n.1, p.144153, 2000. Disponível em< $<$ www.fundaj.gov.br $>$. Acesso em 14/02/2008.

RAPINI, Márcia Siqueira. Interação universidade-empresa no Brasil: evidências do Diretório dos Grupos de Pesquisa do CNPq. Disponível em: <http: //www.scielo.br/scielo.php?pid=S0101-41612007000100008\&script=sci_arttext.>. Estudos Econômicos, vol. 37 no 1 - São Paulo, Jan./Mar 2007. Acesso em: 07/07/2007.

RIBEIRO, Públio Vieira Valadares. Inovação tecnológica e transferência de tecnologia. Disponível em < http://www.mct.gov.br/upd blob/0002/2212.pdf $>$ Acesso em 15/04/2008. 